\title{
TELECONSUTORIA E VIDEOCONFERÊNCIA COMO ESTRATÉGIA DE EDUCAÇÃO PERMANENTE PARA AS EQUIPES DE SAÚDE DA FAMÍLIA
}

Eliane Marina Palhares Guimarães', Solange Cervinho Bicalho Godoy', Lenice de Castro Mendes Villela', Jéssica Diniz Schettini Mafaldo²

${ }^{1}$ Enfermeira. Doutora em Enfermagem. Docente da Escola de Enfermagem da Universidade Federal de Minas Gerais. Belo HorizonteMG-Brasil.

${ }^{2}$ Discente de Enfermagem. Escola de Enfermagem da Universidade Federal de Minas Gerais. Belo Horizonte-MG-Brasil.

RESUMO: A Educação a Distância, através do uso das Tecnologias de Informação e Comunicação superou os limites para capacitação das Equipes de Saúde da Família por meio da educação permanente. O estudo tem como objetivo avaliar a relação existente entre as áreas temáticas solicitadas nas teleconsultorias e os temas propostos para as videoconferências realizadas no período de janeiro de 2008 a dezembro de 2012. Trata-se de um estudo exploratório, com abordagem qualitativa. Foram utilizados dados secundários fornecidos pelo Núcleo de Telessaúde de uma faculdade de Minas Gerais. Foi realizada a análise dos registros de 495 teleconsultorias e 89 videoconferências. O estudo permitiu concluir a aproximação das áreas temáticas das dúvidas descritas nas teleconsultorias com os temas propostos para as videoconferências, indicando que os profissionais utilizam os recursos da Telessaúde para discutirem dúvidas da realidade assistencial. Constata-se a possibilidade de expansão do projeto, com ampliação de suas ações.

DESCRITORES: Telenfermagem; Telessaúde; Consulta remota; Segunda opinião.

\section{TELECONSULTATIONS AND VIDEOCONFERENCING AS A STRATEGY OF PERMANENT EDUCATION FOR FAMILY HEALTH CARE TEAMS}

\begin{abstract}
Through the use of Information and Communication Technologies, distance education exceeded the limits for the qualification of family healthcare teams through permanent education. This study aims to evaluate the existing relation between the thematic areas that are requested in the teleconsultations and the themes proposed for videoconferences conducted between January 2008 and December 2012. It is an exploratory study with a qualitative approach. Secondary data provided by the Telehealth Nucleus of a university in Minas Gerais were used. Records of 495 teleconsultations and 89 videoconferences were analyzed. The study enabled the conclusion of the approximation of the thematic areas of the doubts described in the teleconsultations with the themes proposed for the videoconferences, indicating that the professionals use Telehealth resources to discuss questions about the health care reality. The possibility of expansion of the project and its actions was evidenced. DESCRIPTORS: Telenursing; Telehealth; Remote consultation; Second opinion.
\end{abstract}

\section{TELECONSUTORÍA Y VIDEOCONFERENCIA COMO ESTRATEGIAS DE EDUCACIÓN PERMANENTE PARA LOS EQUIPOS DE SALUD DE LA FAMILIA}

RESUMEN: La Educación a distancia, a través del uso de Tecnologías de Información y Comunicación, ha superado los límites para capacitación de los Equipos de Salud de la Familia por medio de la educación permanente. El estudio tiene como propósito evaluar la relación entre áreas temáticas solicitadas en las teleconsultorías y los temas propuestos para las videoconferencias realizadas en el periodo de enero de 2008 a diciembre de 2012. Es un estudio exploratorio, con abordaje cualitativo. Fueron utilizados datos secundarios fornecidos por el Núcleo de Telesalud de una facultad de Minas Gerais. Fue realizado el análisis de los registros de 495 teleconsultorías y 89 videoconferencias. El estudio posibilitó concluir la aproximación de áreas temáticas de las dudas descriptas en las teleconsultorías con los temas propuestos para las videoconferencias, apuntando que los profesionales utilizan los recursos de la Telesalud para discutir dudas de la realidad asistencial. Se constata la posibilidad de expansión del proyecto, con ampliación de sus acciones. DESCRIPTORES: Telenfermería; Telesalud; Consulta remota; Segunda opinión. 


\section{INTRODUÇÃO}

A Atenção Primária à Saúde (APS) tem utilizado recursos tecnológicos de informação e comunicação no intuito de apoiar os profissionais de saúde, qualificar a assistência e possibilitar mudanças no processo de trabalho da equipe de saúde. A utilização destes recursos está estabelecida pela Portaria $\mathrm{n}^{\circ}$ 2.546, DE 27 DE OUTUBRO DE 2011, que redefine e amplia o Programa Telessaúde Brasil, que passa a ser denominado Programa Nacional Telessaúde Brasil Redes (Telessaúde Brasil Redes) ${ }^{(1)}$. O referido Programa busca uma nova proposta de educação em saúde que incorpore Tecnologias de Comunicação e Informação (TIC's) como meios para a capacitação profissional. O uso de recursos tecnológicos de informação e comunicação possibilita mudanças nas práticas da equipe de saúde e viabiliza ações de apoio à assistência em saúde por meio da educação permanente.

O PNT permite a estruturação de rede integrada para transmissão de dados, sons e imagens, criando ambiente com sustentabilidade para o processo de trabalho das equipes de saúde da Estratégia de Saúde da Família (ESF) e, também, contribui para a consolidação dos princípios do Sistema Único de Saúde (SUS). O PNT visa a educação continuada e a qualificação profissional, por meio da Educação a Distância $(E a D)$ e, em consequência, propicia a permanência dos profissionais nos municípios, especificamente, aqueles mais carentes, além da redução dos deslocamentos desnecessários de pacientes para os grandes centros urbanos.

Na área da saúde a educação a distância está cada vez mais presente sob forma de cursos de aperfeiçoamento, extensão, educação permanente, além de curso de especialização oferecido por instituições de ensino a distância ${ }^{(2)}$. Pode se dizer que

o serviço de educação permanente a distância torna-se ferramenta importante na área da saúde, pois colabora para orientar as práticas e atualizar o profissional em relação a novos saberes ${ }^{(3: 26)}$.

Na enfermagem a educação a distância é um meio cada vez mais utilizado, para estimular o profissional a buscar novos conhecimentos e compartilhar sua experiência prática ${ }^{(4)}$.

No cenário educacional, essas novas ferramentas tecnológicas revolucionaram o acesso à informação e ao conhecimento. A velocidade com que o conhecimento e os saberes tecnológicos se renovam, na área da saúde, torna necessária a atualização permanente dos trabalhadores. A Política de Educação Permanente em Saúde, regulamentada pela Portaria MS/GM $\mathrm{n}^{\circ}$ 1.996, de 20 de agosto de 2007, surge como proposta para viabilizar o processo contínuo de educação, uma vez que, possibilita a aproximação entre o cotidiano do profissional que atua no SUS e as necessidades da população, com o intuito de melhorar a qualidade dos serviços prestados ${ }^{(5)}$.

O processo de Educação Permanente em saúde veio possibilitar melhora das práticas de serviço dos profissionais da equipe de saúde, tornando-os mais envolvidos, interessados, participativos, valorizando o conhecimento dos mesmos e, também, aumentando as oportunidades de aprendizagem no próprio local de trabalho ${ }^{(6)}$.

O sistema de Educação Permanente em Saúde (EPS) pode ser compreendido como um conjunto de alternativas educacionais, articulado, visando ao desenvolvimento de ações voltadas à aprendizagem significativa e para o processo de trabalho.

A educação permanente no trabalho se transforma em um instrumento dinamizador da mudança institucional, facilitando o entendimento, a apreciação e a aceitação do modelo de atenção indicados pelos novos programas, priorizando a busca de opções contextualizadas e integradas para o cuidado da população assistida ${ }^{(7)}$. A partir dessa reflexão, pode-se dizer que

[...] para o setor da saúde, a estética pedagógica da Educação Permanente em Saúde é a de introduzir a experiência da problematização e da invenção de problemas ${ }^{(8: 984)}$.

Com base na importância e na necessidade da educação permanente para a capacitação de profissionais da ESF e com a possibilidade da EaD, foi criado em 2004 o Projeto Telenfermagem, coordenado pela Escola de Enfermagem da Universidade Federal de Minas Gerais, que tem como objetivo capacitar acadêmicos dos cursos 
de graduação e pós-graduação em enfermagem, enfermeiros e trabalhadores de enfermagem, além de profissionais de saúde inseridos nas Unidades Básicas de Saúde (UBS) da Prefeitura de Belo Horizonte $(\mathrm{PBH})$ e de Minas Gerais. No ano de 2008, o Projeto Telenfermagem passou a integrar o Núcleo de Telessaúde (NUTEL) da Faculdade de Medicina da Universidade Federal de Minas Gerais (UFMG), como parte do Programa Nacional de Telessaúde, do qual participam aproximadamente 100 municípios cadastrados pelo programa.

O Projeto Telenfermagem atua em duas linhas de trabalho: teleconsultorias e videoconferências. As videoconferências, que são discussões temáticas, ocorrem quinzenalmente, são agendadas previamente, divulgadas nas unidades participantes e os temas abordados são selecionados pela própria comunidade, a partir de dúvidas da prática diária. Durante as apresentações, dúvidas são respondidas em tempo real pelo conferencista ou por chat, em um processo absolutamente interativo. Estas possuem duração média de 60 minutos, sendo os 30 primeiros minutos para abordagem do tema pelo videoconferencista e o tempo restante para discussão com os profissionais telespectadores.

As teleconsultorias são realizadas por meio do site do Núcleo de Telessaúde de Minas Gerais, no qual os profissionais da área da saúde, como enfermeiros, técnicos em enfermagem, médicos, odontólogos, agentes comunitários de saúde encaminham suas dúvidas para um profissional da equipe de consultores. Os consultores são profissionais especialistas da Escola de Enfermagem da Universidade Federal de Minas Gerais (EE/UFMG) ou Hospital de Clínicas (HC/UFMG) e das unidades de saúde. A resposta à consulta é registrada no próprio sistema e encaminhada diretamente ao solicitante, preferencialmente, em 72 horas.

A teleconsultoria possui duas modalidades: on-line, em que o profissional apresenta e discute um determinado caso clínico com o especialista, em tempo real e a off-line que é utilizada para casos em que o acesso ao profissional especialista é feito por meio do envio da dúvida pelo sistema e a resposta do teleconsultor é enviada dentro do prazo estimado $^{(9)}$.

Pode-se afirmar que o uso da teleconsultoria proporciona grandes benefícios sociais e econômicos para o SUS e para a população, em especial a dos municípios mais distantes dos grandes centros. Primeiro, por propiciar educação a distância de forma continuada, retendo os profissionais nos locais de atuação e, segundo, por reduzir os custos de encaminhamento do paciente ao nível secundário para atendimento especializado.

A teleconsultoria pode possibilitar uma segunda opinião formativa, com o acréscimo de informações e com a antecipação dos acontecimentos mediante uma proposta prática. A segunda opinião formativa é processo interativo que objetiva a transmissão de um conhecimento de forma dirigida para a construção do raciocínio, está centrada na dúvida do profissional requerente, baseando-se no problema encaminhado a um profissional especialista. Desta forma, pode ser aplicada em situações em que um profissional a distância, que necessite do apoio de outro profissional, compartilhe a experiência de especialistas em conhecimento aplicável para a resolução de um problema.

A segunda opinião é solicitada considerando a formação do profissional requerente e na dúvida encaminhado ao profissional especializado. Em seu art. $2^{\circ}$, a Portaria MS/GM 2.546/11 define segunda opinião formativa como

[...] resposta sistematizada, construída com base em revisão bibliográfica, nas melhores evidências científicas e clínicas e no papel ordenador da atenção básica à saúde, a perguntas originadas das teleconsultorias, e selecionadas a partir de critérios de relevância e pertinência em relação às diretrizes do SUS(1:50).

O SUS arca hoje com altos custos de Tratamento Fora de Domicílio relacionados ao transporte de pacientes. Além do custo, muitas vezes o estado grave do paciente a ser transportado coloca em risco a sua própria vida $^{(9)}$. A dificuldade de fixação dos profissionais nos locais distantes dos grandes centros e com recursos limitados é um desafio que pode ser enfrentado estruturalmente pela Telessaúde.

O objetivo do presente estudo é avaliar a relação existente entre as áreas temáticas solicitadas nas teleconsultorias e os temas propostos para as videoconferências realizadas pelo Projeto Telenfermagem, no período de janeiro de 2008 a dezembro de 2012. 


\section{METODOLOGIA}

Trata-se de um estudo exploratório desenvolvido com a finalidade de conhecer um determinado fenômeno, as teleconsultorias e as videoconferências. A pesquisa exploratória consiste no aprofundamento de conceitos preliminares sobre determinada temática, contribuindo assim, para o esclarecimento de questões superficialmente abordadas sobre o assunto $^{(10)}$. O estudo tem como proposta empregar uma metodologia qualitativa, abordagem que possibilita a descrição da complexidade de um determinado problema, análise da interação de certas variáveis, compreensão dos processos dinâmicos vividos pelos grupos sociais e o entendimento das particularidades do comportamento dos indivíduos ${ }^{(11)}$.

Para alcance do objetivo foram utilizados dados secundários fornecidos pelo NUTEL-UFMG e acervo próprio do Projeto Telenfermagem. A pesquisa foi aprovada e sua realização autorizada pela Escola de Enfermagem da Universidade Federal de Minas Gerais e pelo Núcleo de Telessaúde da Faculdade de Medicina da Universidade Federal de Minas Gerais.

Para o estudo foram analisados os registros de 511 teleconsultorias do Programa Nacional de Telessaúde na área da enfermagem, no período de 01 de Janeiro de 2008 a 31 de Dezembro de 2012. A área de conhecimento em enfermagem foi o critério definido para inclusão das teleconsultorias e, para exclusão, foram consideradas as teleconsultorias duplicadas e aquelas usadas como teste do sistema. Do total das 511 teleconsultorias realizadas nesse período, 16 foram excluídas, restando 495 teleconsultorias para análise.

Os dados investigados foram organizados por ano de ocorrência, área temática e descrição da dúvida. Foram classificados em onze áreas temáticas, sendo elas: Tratamento de feridas; Sistematização da assistência de enfermagem; Saúde mental e psiquiátrica; Saúde do adulto e do idoso; Saúde da mulher; Saúde da criança e do adolescente; Processo de trabalho em saúde e na enfermagem; Gestão em saúde e na enfermagem; Fundamentos em enfermagem e Enfermagem. O agrupamento "Enfermagem" foi a primeira área criada no projeto e, nos primeiros meses de desenvolvimento do projeto, permaneceu como única. Em decorrência disto, possui abordagem mais ampla e geral, contendo dúvidas de conteúdos diversos. Todas estas teleconsultorias foram consideradas e compreendem qualquer assunto que não se constituía como uma especialidade dentro das demais áreas temáticas.

No mesmo período foram realizadas 89 videoconferências, que foram organizadas de acordo com o cronograma de desenvolvimento, considerando apenas as videoconferências realizadas, sendo excluídas aquelas que por qualquer motivo foram suspensas ou aquelas que aconteceram apenas para eleição de temas, para o próximo semestre e para avaliação do semestre corrente.

\section{RESULTADOS}

O projeto Telenfermagem realizou no período de 01 de janeiro de 2008 a 31 de dezembro de 2012 um total de 89 videoconferências e 495 teleconsultorias, que foram classificadas conforme demonstrado nas tabela 1 e 2 , a cada ano, em onze áreas temáticas: Tratamento de Feridas, Sistematização da Assistência à Enfermagem (SAE), Saúde Mental e Psiquiátrica, Saúde do Adulto e do Idoso, Saúde da Mulher, Saúde da Criança e do Adolescente, Processo de Trabalho em Saúde e na Enfermagem, Gestão em Saúde e na Enfermagem, Fundamentos em Enfermagem, Educação em Saúde e a área da Enfermagem.

As videoconferências e teleconsultorias realizadas de 2008 a 2012 apresentaram maiores percentuais aquelas referente à área temática de tratamento de feridas, seguidas da saúde da mulher e saúde da criança. A Figura 1 apresenta a porcentagem de teleconsultorias por áreas temáticas realizadas no período de 2008 a 2012. 
Tabela 1 - Relações das videoconferências de acordo com a área temática no período de 2008 - 2012 pelo projeto Telenfermagem/EEUFMG. Belo Horizonte, 2013

\begin{tabular}{|c|c|c|}
\hline Ano & Videoconferências & $\%$ \\
\hline \multirow{8}{*}{2008} & Saúde da criança e do adolescente & 26,4 \\
\hline & Enfermagem & 21 \\
\hline & Saúde da Mulher & 10,5 \\
\hline & Saúde do Adulto e do Idoso & 10,5 \\
\hline & SAE & 10,5 \\
\hline & Tratamento de Feridas & 10,5 \\
\hline & Fundamentos de Enfermagem & 5,3 \\
\hline & Processo de Trabalho em Saúde & 5,3 \\
\hline \multirow{9}{*}{2009} & Enfermagem & 22,1 \\
\hline & Saúde do Adulto e do Idoso & 16,7 \\
\hline & Gestão em Saúde & 11,1 \\
\hline & SAE & 11,1 \\
\hline & Tratamento de Feridas & 11,1 \\
\hline & Saúde da criança e do adolescente & 11,1 \\
\hline & Fundamentos de Enfermagem & 5,6 \\
\hline & Saúde da Mulher & 5,6 \\
\hline & Saúde Mental e Psiquiátrica & 5,6 \\
\hline \multirow{7}{*}{2010} & Saúde da Mulher & 23,55 \\
\hline & Saúde do Adulto e do Idoso & 23,55 \\
\hline & Enfermagem & 17,6 \\
\hline & Saúde da criança e do adolescente & 17,6 \\
\hline & Fundamentos de Enfermagem & 5,9 \\
\hline & Saúde Mental e Psiquiátrica & 5,9 \\
\hline & Tratamento de Feridas & 5,9 \\
\hline \multirow{6}{*}{2011} & Saúde da Mulher & 31,5 \\
\hline & Saúde do Adulto e do Idoso & 25 \\
\hline & Saúde da criança e do adolescente & 18,5 \\
\hline & Educação em Saúde & 12,5 \\
\hline & Tratamento de Feridas & 6,25 \\
\hline & Fundamentos de Enfermagem & 6,25 \\
\hline \multirow{8}{*}{2012} & Saúde da Mulher & 31,5 \\
\hline & Saúde do Adulto e do Idoso & 26,4 \\
\hline & Saúde da criança e do adolescente & 15,8 \\
\hline & Enfermagem & 5,26 \\
\hline & Fundamentos de Enfermagem & 5,26 \\
\hline & Gestão em Saúde & 5,26 \\
\hline & Processo de Trabalho & 5,26 \\
\hline & Saúde Mental e Psiquiátrica & 5,26 \\
\hline
\end{tabular}

Tabela 2 - Relações das teleconferências de acordo com a área temática no período de 2008 - 2012 pelo projeto Telenfermagem/EEUFMG. Belo Horizonte, 2013

\begin{tabular}{|c|c|c|}
\hline Ano & Teleconsultorias & $\%$ \\
\hline \multirow{5}{*}{2008} & Tratamento de Feridas & 50 \\
\hline & Saúde da Mulher & 25 \\
\hline & Saúde da Criança e do Adolescente & 10 \\
\hline & Fundamento de Enfermagem & 10 \\
\hline & Processo de Trabalho em Saúde & 5 \\
\hline \multirow{6}{*}{2009} & Tratamento de Feridas & 62 \\
\hline & Saúde da Mulher & 14 \\
\hline & Enfermagem & 10 \\
\hline & Saúde da criança e do adolescente & 10 \\
\hline & Educação em saúde & 2 \\
\hline & Saúde Mental e Psiquiátrica & 2 \\
\hline \multirow{10}{*}{2010} & Tratamento de Feridas & 32 \\
\hline & Saúde da criança e do adolescente & 18 \\
\hline & Saúde da Mulher & 11 \\
\hline & Fundamentos de Enfermagem & 9 \\
\hline & SAE & 9 \\
\hline & Enfermagem & 7 \\
\hline & Educação em Saúde & 5 \\
\hline & Saúde do Adulto e do Idoso & 5 \\
\hline & Saúde Mental e Psiquiátrica & 2 \\
\hline & Gestão em Saúde e na Enfermagem & 2 \\
\hline \multirow{9}{*}{2011} & Tratamento de Feridas & 45 \\
\hline & Saúde da Mulher & 24 \\
\hline & Saúde do Adulto e do Idoso & 9 \\
\hline & Saúde da criança e do adolescente & 5 \\
\hline & Fundamentos de Enfermagem & 5 \\
\hline & SAE & 4 \\
\hline & Educação em Saúde & 4 \\
\hline & Saúde Mental e Psiquiátrica & 2 \\
\hline & Processo de Trabalho em Saúde & 2 \\
\hline \multirow{11}{*}{2012} & Tratamento de Feridas & 30 \\
\hline & Saúde da Mulher & 22 \\
\hline & Saúde da Criança e do Adolescente & 12 \\
\hline & Enfermagem & 11 \\
\hline & Educação em Saúde & 6,5 \\
\hline & Saúde do Adulto e do Idoso & 6,5 \\
\hline & SAE & 5 \\
\hline & Saúde Mental e Psiquiátrica & 4 \\
\hline & Gestão em Saúde e na Enfermagem & 1 \\
\hline & Processo de Trabalho em Saúde & 1 \\
\hline & Fundamento de Enfermagem & 1 \\
\hline
\end{tabular}




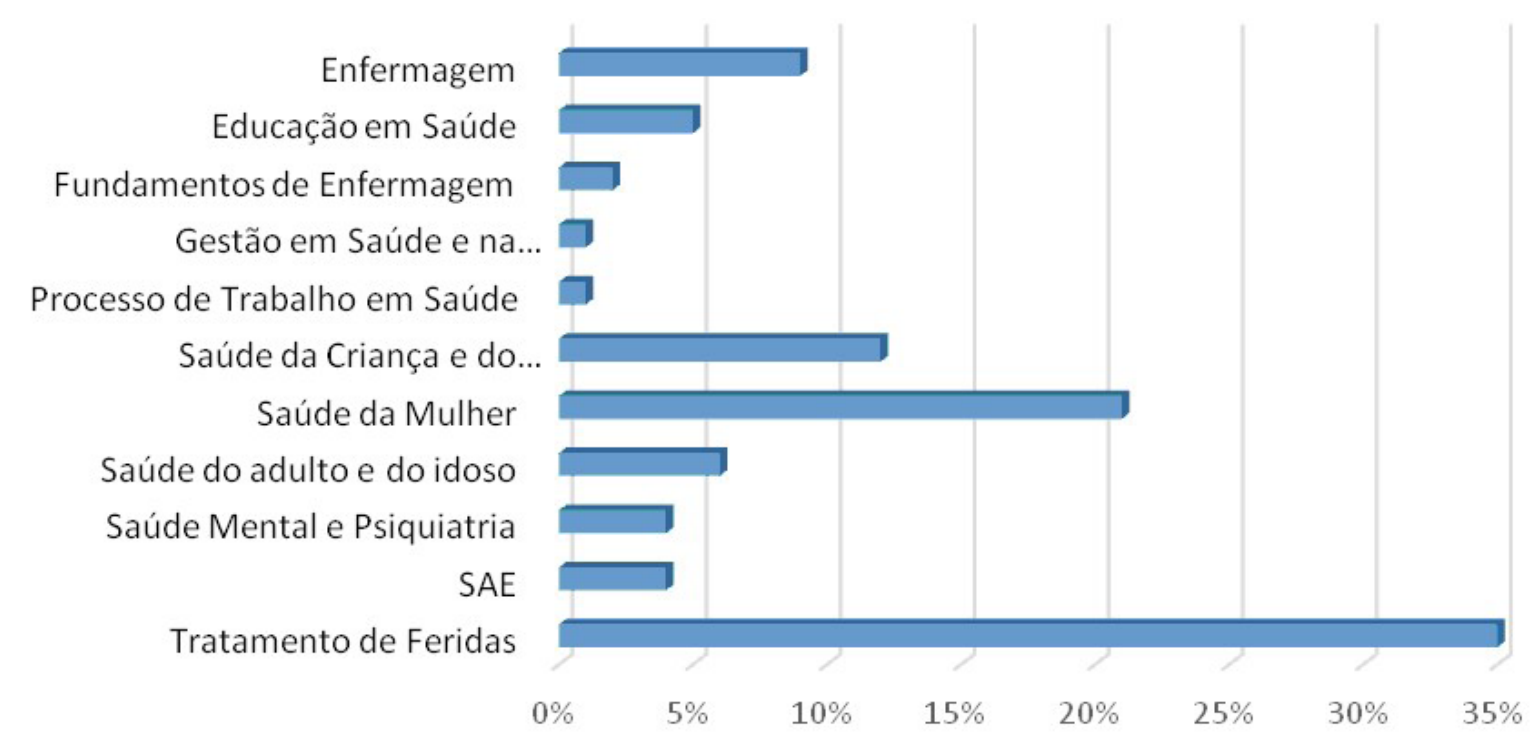

Tabela 1 - Relações das videoconferências de acordo com a área temática no período de 2008 - 2012 pelo projeto Telenfermagem/EEUFMG. Belo Horizonte, 2013

\section{DISCUSSÃO}

No ano de 2008 foram realizadas 19 videoconferências e 20 teleconsultorias. O tema mais solicitado nas teleconsultorias foi tratamento de feridas, gerando uma segunda opinião formativa para dúvidas de tratamento de úlcera venosa, ferida que acomete principalmente a população adulta e idosa.

Foi constatado ainda que no conjunto das teleconsultorias sobre tratamento de feridas, muitas delas apresentaram apenas a descrição da ferida em si, sem detalhes do caso clínico e, também, sem dados sobre a assistência prestada. Nestes casos, como não apresentavam a formulação de uma dúvida específica, o teleconsultor muitas vezes precisou devolver a questão ao profissional, solicitando maiores detalhes e reenvio da teleconsultoria.

Neste mesmo ano, em relação às videoconferências, os assuntos abordados com maior frequência foram relacionados à Saúde da Mulher e Saúde da Criança e do Adolescente, mostrando que não houve uma aproximação temática entre as demandas da unidade básica de saúde, retratadas pelas solicitações pela teleconsultoria, ou seja, a área de feridas. Isto pode supor que não há uma vinculação entre as videoconferências e as necessidades cotidianas dos profissionais de saúde.
No ano de 2009, foram realizadas 18 videoconferências e 42 teleconsultorias. $\mathrm{O}$ tema de maior demanda para as videoconferências foi referente à Enfermagem, abordando uma maior variedade de assuntos. Nesse ano, como no ano anterior, o tema Tratamento de Feridas foi o mais demandado para as teleconsultorias, assim como as dúvidas apresentadas sobre tipos de cobertura e sua indicação.

Analisando as videoconferências ocorridas em 2009, os temas mais demandados foram Enfermagem e Saúde do Adulto e do Idoso, o que demonstra uma aproximação temática entre as teleconsultorias e as videoconferências. Compreende-se que as dúvidas em relação ao tratamento de feridas nas teleconsultorias são mais frequentes na faixa etária de adulto e idoso, e os temas tratados nas videoconferências concentraram-se na área da enfermagem em geral, trabalhando diversos assuntos, incluindo também questões relacionadas ao tratamento de feridas.

Tanto no ano de 2008 como em 2009, o tema Tratamento de Feridas foi o assunto mais demandado para as Teleconsultorias, o que permite afirmar que as dúvidas são persistentes e se repetem e, que os profissionais se remetem a situações advindas da demanda assistencial diária. Corroborando com esta afirmativa sabe-se que nas UBS a atividade demandada com mais frequência é o curativo de feridas, sejam elas cirúrgicas ou não. 
No ano de 2010 foram realizadas 17 videoconferências e 44 teleconsultorias. As temáticas Saúde da mulher e Saúde do adulto e do Idoso destacaram-se entre os temas mais solicitados nas videoconferências. O tema Tratamento de Feridas continuou sendo um dos mais solicitados e de maior demanda nas teleconsultorias, porém persistiram as falhas na descrição do caso e da dúvida propriamente dita. Observa-se também que o tema Saúde da mulher apareceu em segundo lugar nas solicitações para as teleconsultorias.

Percebe-se que Saúde da mulher foi um tema de grande solicitação nas duas atividades do projeto, destacando a aproximação temática das duas atividades desenvolvidas e reafirmando a relevância do assunto na demanda assistencial. Em relação ao tema Tratamento de feridas, as dúvidas mais frequentes abordam lesões do tipo úlcera venosa e lesões que acometem membros inferiores de pacientes portadores de diabetes, que por sua vez, estão relacionadas com a alta demanda do tema Saúde do adulto e do idoso, nas videoconferências.

No ano de 2011 foram realizadas 16 videoconferências e 55 teleconsultorias. Nas videoconferências realizadas houve um aumento significativo na abordagem do tema Saúde da mulher e Saúde da Criança e do Adolescente. Fato que se manteve no ano de 2012, como descrito a seguir, e que pode ser explicado pela implantação do Programa "Rede Cegonha" pelo Ministério da Saúde, que propõe estratégias para implantação de uma rede de cuidados para assegurar às mulheres o direito ao planejamento reprodutivo e a atenção humanizada à gravidez, ao parto e ao puerpério, bem como assegurar às crianças o direito ao nascimento seguro e ao crescimento e desenvolvimento saudáveis ${ }^{(12)}$. As dúvidas mais frequentes nas teleconsultorias abordam questões relacionadas ao exame preventivo e ao exame citopatológico e, nas videoconferências abordaram questões mais amplas, como prénatal, risco de gravidez na adolescência e exame citopatológico.

No ano de 2012 foram realizadas 19 videoconferências e 334 teleconsultorias. Neste ano, todas as 11 áreas temáticas foram solicitadas, mostrando o crescimento das ações desenvolvidas pelo projeto, além do interesse dos profissionais acerca de uma amplitude maior de temas.
Um fator comum observado no decorrer destes cinco anos, é que não houve solicitação da área temática Gestão em Saúde, tanto nas teleconsultorias como nas videoconferências. Entende-se que os encaminhamentos das dúvidas são feitos pelos profissionais de saúde que não estão atuando na gestão da unidade, propriamente dita, o que pode explicar a pouca demanda para a área.

Saúde da Mulher foi a área mais solicitada nas videoconferências e a segunda maior demanda para as teleconsultorias e, Saúde do adulto e do idoso foi o segundo tema mais solicitado nas videoconferências. O tema mais demandado nas teleconsultorias foi tratamento de feridas, mostrando a aproximação temática entre as atividades desenvolvidas pelo projeto. É importante destacar que era esperada uma maior demanda da Saúde do Adulto e do Idoso, considerando a tendência mundial de envelhecimento da população e aumento da expectativa de vida, bem como o controle das doenças crônico degenerativas. Além disto, o grupo etário adulto e idoso, quando analisado no conjunto, compreende grande parte da população que busca atendimento na atenção básica, sendo maior, o grupo de idosos.

Após análise dos dados é possível constatar que nos cinco anos de estudos a área temática Tratamento de feridas foi a mais solicitada nas teleconsultorias e os problemas encontrados para resposta aos profissionais no decorrer destes anos se repetiram, destacando-se entre eles, a forma como as dúvidas são apresentadas ao teleconsultor, com dados insuficientes para se compreender o caso enviado. É comum o relato de dúvidas sem descrição da história do paciente, comorbidades, resultados de exames laboratoriais e a descrição dos cuidados que foram prestados até o momento da solicitação de uma segunda opinião.

Diante do exposto compreende-se ser necessária a sistematização na descrição das teleconsultorias para serem enviadas ao teleconsultor de forma clara, possibilitando a emissão de um parecer técnico. Em se tratando de um caso clínico devem-se incluir as principais informações, iniciando com os dados do paciente: iniciais do nome, idade, sexo, profissão. Em relação ao caso clínico em si, apresentar o diagnóstico; o tratamento proposto e o tratamento realizado e finalizar apresentando a evolução do 
cliente com a formulação da pergunta objetiva sobre o caso clínico.

\section{CONSIDERAÇÕES FINAIS}

As teleconsultorias e as videoconferências, de janeiro de 2008 a dezembro de 2012, apresentam uma aproximação das áreas temáticas das dúvidas descritas nas teleconsultorias e os temas propostos para as videoconferências, indicando que os profissionais utilizam os recursos da Telessaúde para discutirem a realidade assistencial vivenciada nas UBS. Os resultados apontam que a área temática Tratamento de Feridas foi a mais solicitada pelas Teleconsultorias e apareceram com destaque nos temas abordados nas videoconferências. A partir de 2011, com a implementação da Rede Cegonha, a temática Saúde da Mulher e Saúde da Criança e do Adolescente foram os principais temas abordados nas videoconferências e apresentaram crescimento nas solicitações das teleconsultorias.

É possível concluir que o estudo alcançou seu objetivo, indicando a existência da relação entre as áreas temáticas solicitadas nas teleconsultorias e os temas propostos para as videoconferências realizadas pelo Projeto Telenfermagem, no período de janeiro de 2008 a dezembro de 2012.

Os resultados do projeto ressaltam a importância do uso das TIC's nas iniciativas de Educação a Distância, como estratégia metodológica para a educação permanente das ESF em municípios do interior de MG. Além disto, apontam para a necessidade de realização de outros estudos para aprofundamento da temática, tal como a verificação do impacto na melhoria da assistência a partir das ações de capacitação desenvolvidas.

Com base nestes resultados, comprova-se a eficiência das atividades desenvolvidas pelo projeto para a capacitação dos profissionais de saúde que atuam nas UBS e constata-se a possibilidade de expansão do projeto, com ampliação de suas ações. Sugere-se a realização de videoconferências originadas nos municípios participantes do projeto, para apresentação e discussão de um caso clínico de interesse paras as ESF; analisar as dúvidas mais frequentes entre os municípios e promover discussões temáticas entre esses, se possível fazendo com que eles sejam referências entre si.

É possível também, a partir das dúvidas mais frequentes, investir na formação acadêmicas dos alunos de graduação, uma vez que grande parte dos teleconsultores fazem parte da academia, podendo desenvolver projetos e ações que aprofunde na discussão das principais demandas da atenção básica. Além destas contribuições, uma ação que traria grande impacto e atenderia a demanda explícita das ESF é a disponibilização do acervo do material produzido pelo projeto, a partir da gravação das videoconferências e edição das mesmas como material instrucional, com acesso livre pelas ESF no Portal do Programa Nacional de Telessaúde. Esta iniciativa se encontra em andamento e alguns dos materiais produzidos pelo projeto estão disponíveis no Repositório de Material Didático da UFMG.

\section{REFERENCIAS}

1. BRASIL. Portaria MS/GM no 2546, de 27 de outubro de 2011. Redefine e amplia o Programa Telessaúde Brasil, que passa a ser denominado Programa Nacional Telessaúde Brasil Redes (Telessaúde Brasil Redes). Diário Oficial da União, Brasília, DF, 2011. No 243/2011, secção I, p.50.

2. Rodrigues RCV, Peres HHC. Panorama brasileiro do ensino de enfermagem a distância. Rev. esc. enferm. USP. 2007;42(2):298-304.

3. Faria MGA, David HMSL. Telessaúde Brasil - núcleo Rio de Janeiro: a educação permanente no trabalho de enfermeiros da atenção básica [dissertação]. Rio de Janeiro (RJ): Faculdade de Enfermagem da Universidade do Estado do Rio de Janeiro; 2010.

4. Oliveira MAN. Educação à distância como estratégia para educação permanente em saúde: possibilidades e desafios. Rev. bras. enferm. 2007;60(5):585-9.

5. Ministério da Saúde (BR). Portaria n. 1996, de 20 de agosto de 2007. Dispõe sobre as diretrizes para a implementação da Política Nacional de Educação Permanente em Saúde e dá outras providências. Diário Oficial da União, Brasília, 22 ago. 2007. Seção 1:1.

6. Carotta F, Kawamura D, Salazar J. Educação permanente em saúde: uma estratégia de gestão para pensar, refletir e construir práticas educativas e processos de trabalhos. Saúde soc. 2009;18(1):48-51.

7. Davini MC. Enfoques, Problemas e Perspectivas na Educação Permanente dos Recursos Humanos de 
Saúde. Política Nacional de Educação Permanente em Saúde. Brasília: Ministério da Saúde, 2009. Série pacto pela saúde. 19: 39-59.

8. Ceccim RB. Educação Permanente em Saúde: descentralização e disseminação de capacidade pedagógica na saúde. Ciênc. saúde coletiva. [Internet] 2005;10(4) [acesso em 28 jan 2014]. Disponível: http:// dx.doi.org/10.1590/S1413-81232005000400020

9. Santos AF, Souza C, Alves HJ, Santos SM. Telessaúde: um instrumento de suporte assistencial e educação permanente. Belo Horizonte: Editora UFMG; 2006.

10. Gil AC. Métodos e técnicas de pesquisa social. SP:Atlas. 1999:5.

11. Triviños ANS. Introdução à pesquisa em ciências sociais: a pesquisa qualitativa em educação. SP: Atlas. 1987.

12. Ministério da Saúde (BR). Portaria n. 2.395, de 11 de outubro de 2011. Organiza o Componente Hospitalar da Rede de Atenção às Urgências no âmbito do Sistema Único de Saúde (SUS). Diário Oficial da União, Brasília, 13 out. 2011. Seção 1:1. 